\title{
Qualidade de vida em pacientes submetidos ao tratamento quimioterápico
}

\author{
Quality of life in patients undergoing chemotherapy \\ Taise Karine Cutrim Sousa ${ }^{1}$; Cinara Regina Aragão Vieira Monteiro²
}

\begin{abstract}
Resumo: Introdução: O câncer é uma patologia caracterizada pela mutação genética no DNA da célula e proliferação desordenada. Entre as modalidades terapêuticas das neoplasias, a quimioterapia possui vasta utilização, porém, o tratamento com as drogas antineoplásicas pode apresentar várias alterações afetando a qualidade de vida do paciente oncológico. Objetivo: avaliar a qualidade de vida de pacientes submetidos ao tratamento quimioterápico. Materiais e métodos: Foi aplicado um questionário em 76 pacientes, atendidos em um hospital de referência oncológica, que contemplou dados clínicos e sociodemográficos e o SF-36 para avaliação da qualidade de vida. O estudo foi aprovado pelo comitê de ética da Universidade Ceuma. Os dados foram avaliados pelo programa NCSS 11 (2016) e o teste de qui-quadrado de independência $\left(\chi^{2}\right)$. Resultados: A amostra foi composta por $52,6 \%$ de pacientes do sexo feminino, o grupo etário mais acometido foi de 50 a 59 anos $(34,2 \%)$, prevalência da raça branca $(40,8 \%)$. Os domínios de qualidade de vida que apresentaram menores escores foram limitação por aspectos físicos $(78,9 \%)$ e emocionais $(98,7 \%)$. Conclusão: Os efeitos colaterais após a quimioterapia tiveram grande influência na qualidade de vida dos pacientes evidenciando a importância da reabilitação globalizada dos pacientes, durante todo o período de tratamento quimioterápico.
\end{abstract}

Palavras-chave: câncer; quimioterapia; qualidade de vida.

Abstract: Introduction: Cancer is a pathology characterized by genetic mutation in the cell's DNA and disordered proliferation. Among the therapeutical modalities of neoplasias, chemotherapy is widely used, however, treatment with antineoplastic drugs may present several alterations affecting the quality of life of cancer patients. Objective: to evaluate the quality of life of patients undergoing chemotherapy. Materials and methods: A questionnaire was applied in 76 patients, attended at a hospital oncological reference, which included clinical and sociodemographic data and the SF-36 to evaluate the quality of life. The study was approved by the ethics committee of the Ceuma University. The data were evaluated by the NCSS program 11 (2016) and the chi-square test of independence $(\chi 2)$. Results: The sample consisted of $52.6 \%$ female patients, the most affected age group was 50 to 59 years old $(34.2 \%)$, white prevalence $(40.8 \%)$. Quality of life domains that presented lower scores were limited by physical $(78.9 \%)$ and emotional aspects (98.7\%). Conclusion: The side effects after chemotherapy had a great influence on patients' quality of life, evidencing the importance of the globalized rehabilitation of the patients, during the whole period of chemotherapy treatment.

Keywords: cancer; chemotherapy; quality of life.

1- Discente do curso de Fisioterapia - Universidade CEUMA, São Luís - Maranhão, Brasil.

2- Preceptora de estágio hospitalar do curso de Fisioterapia - Universidade CEUMA, São Luís - Maranhão, Brasil. 


\section{Introdução}

O câncer é uma desordem patológica, da qual uma célula anômala é modificada por mutação genética do DNA celular. Essa célula cancerígena passa pelo processo de mitose que começa a multiplicar-se de forma anormal. Após a proliferação, as mesmas atingem características invasivas produzindo metástases ${ }^{1}$.

As neoplasias têm se tornado um dos principais problemas de saúde mundial, visto que, a incidência da doença tem aumentado drasticamente, apresentando elevados índices de mortalidade em ambos os sexos. Estima-se que no biênio 2018-2019 ocorram 600 mil novos casos de câncer no Brasil a cada ano, com predomínio na incidência dos cânceres de próstata, pulmão, mama feminina, cólon e reto. As regiões Sul e Sudeste concentram $70 \%$ dos novos casos no país (INCA, 2018).

O tratamento das neoplasias consiste basicamente em cirurgia, radioterapia, quimioterapia e hormonioterapia. No entanto, as drogas manipuladas na quimioterapia têm ação tanto em células neoplásicas como também nas células normais, ocasionando uma série de efeitos colaterais ao paciente. Os efeitos podem surgir precocemente (náuseas, vômitos e artralgias), imediatos (mucosite, mielossupressão, plaquetopenia e anemia), tardiamente (alopecia e imunossupressão) ultratardiamente (infertilidade, sequelas no sistema nervoso central e fibrose) ${ }^{3,4}$.

As drogas mais utilizadas na quimioterapia antineoplásica ou antiblástica são os alquilantes polifuncionais que agem nos ciclos celulares, os antimetabólitos que bloqueiam a multiplicação e funções normais da célula, os antibióticos antitumorais possuem em comum anéis insaturados que possibilizam a incorporação de excesso de elétrons e a consequente produção de radicais livres reativos e os inibidores mitóticos que impedem a mitose na metáfase, ou seja, a interrupção da divisão celular ${ }^{3}$.

A alta incidência do câncer junto com 0 índice de cura insatisfatória estabelece esforços redobrados de uma equipe multidisciplinar, na compreensão da necessidade da inclusão de instrumentos para avaliar a qualidade de vida dos pacientes. Raramente há doenças crônicas que provocam tantos sentimentos negativos: o medo do diagnóstico, da cirurgia, a incerteza do prognóstico e reincidência, efeitos da radioterapia e quimioterapia, o sofrer pela dor e o enfrentamento da possibilidade da morte ${ }^{5}$.

O diagnóstico de câncer traz mudanças importantes no modo de viver, com alterações físicas e emocionais devido ao desconforto, dor, desfiguração, dependência e perda da autoestima ${ }^{6}$.

Este estudo objetivou avaliar a qualidade de vida de pacientes submetidos ao tratamento quimioterápico e contribuiu para identificar quais aspectos são mais afetados pela quimioterapia que interferem na manutenção da funcionalidade. 
Materiais e métodos

Trata-se de um estudo
epidemiológico, descritivo, observacional e transversal, realizado no hospital Tarquínio Lopes Filho, localizado em São Luís, Maranhão. A amostra foi de conveniência, composta por 76 . Foram adotados os seguintes critérios de inclusão: diagnóstico de câncer que estivessem em tratamento quimioterápico, idade igual ou superior a 18 anos atendidos e acompanhados no hospital e que aceitaram participar da pesquisa assinando o TCLE, e como critérios de exclusão adotouse: pacientes sem condições clínicas de responder a pesquisa.

A coleta de dados foi feita através da aplicação de um questionário, que contemplava dados sócio - demográficos, dados clínicos e para avaliação da qualidade de vida, foi feita através do questionário de qualidade de vida Short-form 36 (SF-36), desenvolvido em 1992 por Ware e Sherbourne e validado no Brasil por Ciconelli ${ }^{7}$, que contemplava 8 domínios: dor, saúde mental, vitalidade, aspectos físicos, capacidade funcional, estado geral, aspectos sociais e aspectos emocionais, nos quais foram atribuídos valores que variavam de 0 a 100 , onde $0=$ pior e $100=$ melhor para cada domínio.

Os dados foram avaliados pelo programa NCSS $11^{8}$. Inicialmente foi feito a estatística descritivas de todos as variáveis através de gráficos e tabelas de frequências. $E$ estimativas de média, desviopadrão, máximo e mínimo das variáveis numéricas e das variáveis ordinais (qualidade de vida) estimouse as medianas e o intervalo interquartil. Posteriormente, para se avaliar associação entre o tipo de tratamento, os tipos de câncer e as variáveis da qualidade de vida (SF36) fez o teste de qui-quadrado de independência $\left(\chi^{2}\right)$. O nível de significância para se rejeitar a hipótese de nulidade será de $5 \%$, ou seja, considerar-se-á como estatisticamente significante um valor de $p<0,05$.

Esta pesquisa foi aprovada pelo comitê de ética da Universidade Ceuma, através do parecer $\mathrm{n}^{\circ}$ 2.145.267.

\section{Resultados}

A amostra da pesquisa foi composta por $52,6 \%$ de pacientes do sexo feminino, o grupo etário mais acometido foi de 50 a 59 anos (34,2\%), com prevalência da raça branca (40,8\%). Com relação à profissão e escolaridade, 36,8\% eram lavradores e $42,1 \%$ cursaram até 0 ensino fundamental (Tabela 1).

Os tipos de câncer mais prevalentes nos pacientes foram no sistema digestivo $(34,2 \%)$, sistema reprodutivo $(15,8 \%)$ e sistema endócrino (11,8\%) (Figura 1). A medicação antineoplásica que foi mais utilizada no tratamento quimioterápico foi a Cisplatina (14,5\%) (Figura 2).

$\mathrm{Na}$ pesquisa da qualidade de vida (SF-36) do paciente observouse que a limitação por aspectos físicos e emocionais foram os domínios mais afetados após o tratamento com a quimioterapia. 
Tabela 1: Características sócio - demográficas da população estudada.

\begin{tabular}{lc}
\hline \multicolumn{1}{c}{ Variáveis } & $\mathbf{N}(\%)$ \\
\hline Sexo & \\
Masculino & $36(47,4)$ \\
Feminino & $40(52,6)$ \\
Faixa etária & \\
$<30$ & $7(9,2)$ \\
$30-39$ & $5(6,6)$ \\
$40-49$ & $12(15,8)$ \\
$50-59$ & $26(34,2)$ \\
$60-69$ & $15(19,7)$ \\
$70-79$ & $7(9,2)$ \\
$>79$ & $3(3,9)$ \\
Ignorada & $1(1,3)$ \\
Cor/raça & \\
Branca & $31(40,8)$ \\
Parda & $29(38,2)$ \\
Negra & $16(21,1)$ \\
Escolaridade & \\
Analfabeto & $9(11,8)$ \\
Ens.Fundam. Incompleto & $32(42,1)$ \\
Ens. Fundam. Completo & $6(7,9)$ \\
Ens. Médio Completo & $24(31,6)$ \\
Ens. Superior Completo & $5(6,6)$ \\
Atividade profissional & \\
Lavrador & $28(36,8)$ \\
Professor & $7(9,2)$ \\
Autônomo & $5(6,6)$ \\
Motorista & $4(5,3)$ \\
Chefe de cozinha & $3(3,9)$ \\
Pescador & $3(3,9)$ \\
Costureira & $2(2,6)$ \\
Doméstica & $2(2,6)$ \\
Dona de casa & $2(2,6)$ \\
Aposentado & $2(2,6)$ \\
18 ocupações (1x) & $18(23,7)$ \\
\hline Total & $76(100)$ \\
\hline & \\
\hline & \\
\hline & \\
\hline &
\end{tabular}




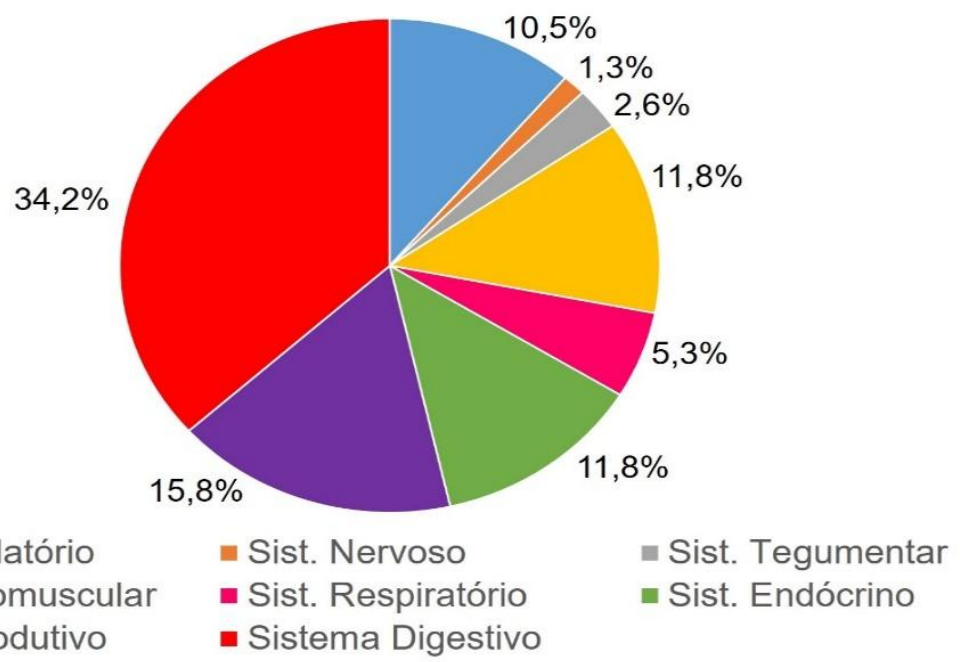

Figura 1: Distribuição percentual dos tipos de câncer da amostra estudada.

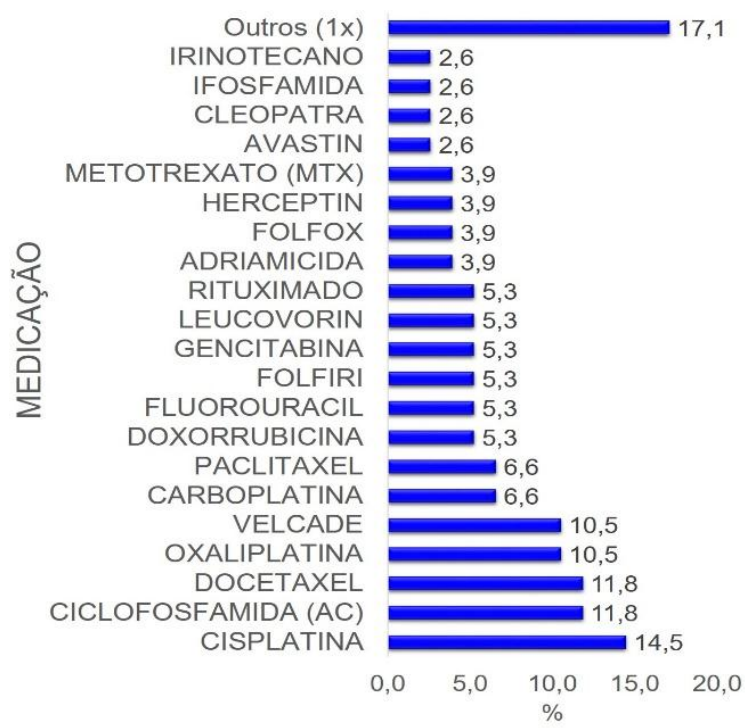

Figura 2: Drogas antineoplásicas mais usadas para tratamento quimioterápico da amostra estudada. 
Tabela 3. Resultados da avaliação da qualidade de vida através do questionário SF 36

\begin{tabular}{lc}
\hline \multicolumn{1}{c}{ SF-36 } & $\mathbf{N}(\%)$ \\
\hline Capacidade funcional & \\
$5-20$ & $18(23,7)$ \\
$21-40$ & $8(10,5)$ \\
$41-60$ & $19(25,0)$ \\
$61-80$ & $14(18,4)$ \\
$81-100$ & $17(22,4)$ \\
\hline Saúde mental & \\
$0-20$ & $3(3,9)$ \\
$21-40$ & $3(3,9)$ \\
$41-60$ & $12(15,8)$ \\
$61-80$ & $19(25,0)$ \\
$81-100$ & $39(51,3)$ \\
\hline Limitação por aspectos físicos & \\
0 & $60(78,9)$ \\
25 & $13(17,1)$ \\
50 & $1(1,3)$ \\
75 & $2(2,6)$ \\
\hline Limitação por aspectos emocionais & \\
33,3 & $75(98,7)$ \\
100,0 & $1(1,3)$ \\
\hline Aspectos sociais & \\
$<50$ & $12(15,8)$ \\
$50-60$ & $5(6,6)$ \\
$60-80$ & $27(35,5)$ \\
$80-100$ & $32(42,1)$ \\
\hline Dor & \\
0 & $2(2,6)$ \\
$10-39$ & $18(23,7)$ \\
$40-69$ & $22(28,9)$ \\
$70-100$ & $34(44,7)$ \\
\hline Estado geral de saúde & \\
$<26$ & $2(2,6)$ \\
$26-50$ & $11(14,5)$ \\
$51-75$ & $16(21,1)$ \\
$>75$ & $47(61,8)$ \\
\hline Vitalidade & \\
$<26$ & $6(7,9)$ \\
$26-50$ & $5(6,6)$ \\
$51-75$ & $34(44,7)$ \\
$>75$ & $31(40,8)$ \\
\hline & \\
\hline & \\
\hline & \\
\hline &
\end{tabular}




\section{Discussão}

Os principais achados deste estudo foram as alterações na qualidade de vida nos aspectos físicos e emocionais, além do comprometimento da funcionalidade do paciente sujeito ao tratamento quimioterápico. As drogas antineoplásicas mais utilizadas foram a cisplatina e ciclofosfamida que pertencem à classe dos alquilantes polifuncionais.

Os alquilantes polifuncionais inibem da multiplicação celular. Afetam as células em todas as fases do ciclo celular. Raramente produzem efeito clínico efetivo sem a combinação com outros agentes fase-específicos do ciclo celular ${ }^{9}$.

A cisplatina é administrada por infusão endovenosa contínua e corresponde a uma droga ciclocelular não específica. Pode provocar efeitos colaterais como a mielodepressão, náuseas e vômitos severos, neuropatia periférica, ototoxicidade, nefrotoxicidade e fadiga ${ }^{10}$.

Sawada et a ${ }^{11}$ realizaram um estudo com 30 pacientes, onde 5 $(16,7 \%)$ apresentaram sintomas gastrointestinais, $3 \quad(10 \%)$ queixaram-se de sintomas físicos como: calor, sede, mal-estar geral, fraqueza, sonolência, insônia, tontura, dor, tremor e coceira, apenas $1 \quad(3,3 \%)$ queixou-se de problemas emocionais. Cerca de $23,3 \%$ pacientes relataram sintomas gastrointestinais e físicos, 13,3\% apresentaram problemas gastrointestinais, físicos e emocionais e $33,3 \%$ estiveram assintomáticos. A mesma pesquisa retratou ainda que os pacientes consideravam o seu estado geral de saúde e qualidade de vida razoável, além disso, encontravam-se preocupados, nervosos e irritados. No entanto as funções físicas, cognitivas, social e desempenho de papel em um grau satisfatório.

Estudos descreveram que a evolução tecnológica dos agentes quimioterápicos levou uma redução nos efeitos colaterais, contribuindo para uma melhor eficácia no tratamento da doença e com relação direta em uma menor vinculação do paciente aos transtornos gerados pelo tratamento ${ }^{12}$.

Uma pesquisa realizada com 438 mulheres com média de idade entre 50 e 62 anos em tratamento quimioterápico, observou que o tipo tumoral ginecológico foi 0 responsável pela pior capacidade funcional apresentada pelas pacientes, seguido do câncer de mama. No que diz respeito a terapêutica utilizada, as pacientes que utilizaram a quimioterapia em associação com outras terapêuticas apresentaram a pior capacidade funcional ${ }^{13}$.

Silva et al. ${ }^{14}$, avaliaram a associação entre qualidade de vida em função das drogas antineoplásicas. Observou-se que existem diferenças na escala de qualidade de vida global, sintomatologia, efeitos físicos da quimioterapia e preocupações com a queda de cabelo. As pacientes em uso do protocolo docetaxel apresentaram melhores resultados na escala qualidade de vida, opondo-se quanto ao uso do paclitaxel, no qual é uma droga antineoplásica, usada no tratamento de câncer de mama, ovário e outros tumores. Dentre os efeitos colaterais destacam-se mielossupressão e neuropatia periférica ${ }^{15}$. 


\section{Conclusão}

\begin{abstract}
Os efeitos colaterais após a quimioterapia tiveram grande influência na qualidade de vida dos pacientes, principalmente nas limitações por aspectos físicos e emocionais, visto que estes podem repercutir na capacidade funcional.

Sendo assim, evidenciou-se a necessidade de assistência multidisciplinar, com ênfase na fisioterapia de manutenção durante todo o tratamento oncológico, visando uma reabilitação globalizada.
\end{abstract}

\section{Agradecimentos}

Primeiramente sou grata a Deus pela vida, saúde e força para superar todas as dificuldades, à toda minha família, principalmente aos meus pais por todo apoio e incentivo nas horas difíceis de desânimo e cansaço, à minha professora orientadora Cinara Aragão pela dedicação, confiança, paciência e por me proporcionar tanto conhecimento. E por fim, não menos importante às minhas amigas Aline, Hellen e Carol que não mediram esforços para me ajudar neste trabalho e por estarem sempre comigo. A todos vocês, minha eterna gratidão!

\section{Referências}

1. Smeltzer SC., Bare BG., Hinkle JL., Cheever KH. Tratado de Enfermagem Médico-Cirúrgica. 11 ed. Rio de Janeiro (RJ): Guanabara Koogan, 2008.

2. INCA - Instituto Nacional do Câncer: Incidência de câncer no Brasil estimativa 2018. [Internet]. Disponível em:http://www.inca.gov.br/estimativa /2018/introducao.asp. Acesso em 07/06/2018.

3. INCA - Instituto Nacional do Câncer: Dia Nacional de Combate ao Câncer 2016. [Internet]. Disponível em: <http://www.inca.gov.br/wcm/dncc/2 015/dia-nacional-combatecancer.asp>. Acesso em 03/04/2017.

4. Kabak VY., Tas N., Ekinci Y., Uysal SA., Düger T. Investigation on The Physical and Functional Needs in Adult Cancer Patients Consulted to Physioterapy and Rehabilitation. Turkish Journal of Oncology. 2016; 31: 104-108.

5. Franzi AS., Silva PG. Qualidade de vida de pacientes submetidos à quimioterapia. Revista Brasileira de Cancerologia. 2003, 49: 153-158.

6. Michelone APC., Santos VLCG. Quality of life of cancer patients with and without an ostomy. Rev Latinoam Enfermagem. 2004; 12: 34-47.

7. Ciconelli RM., Campolina AG., Bortoluzzo AB., Ferraz MB. Validação da versão brasileira do questionário genérico de qualidade de vida short-form 6 dimensions (SF -6D Brasil). Ciência e Saúde Coletiva. 2011; 16: 3103-3110.

8. NCSS 11 Statistical Software (2016). NCSS, LLC. Kaysville, Utah, USA, ncss.com/software/ncss.

9. Schulze MM. Tratamento Quimioterápico em Pacientes Oncológicos. Rev. Bras. Oncologia Clínica. 2007; 4: 17-23.

10. Bonassa EMA. Enfermagem em terapêutica oncológica. 2a ed. São Paulo: Atheneu; 2000.

11. Sawada NO., Nicolussi AC., Okino L., Cardozo FMC., Zago MMF. Avaliação da qualidade de vida de 
pacientes com câncer submetidos à quimioterapia. Revista Escola Enfermagem USP. 2008; 43: 581587.

12. Massarenti M., Silva CSR., Modenez SS. Avaliação da presença de acompanhante durante as sessões de quimioterapia no Instituto Brasileiro de Controle do Câncer (IBCC). O Mundo da Saúde. 2009; 33: 31-34.

13. Silva SR., Haas VJ., Soares MBO., Mendes LC., Elias TC. Influência de variáveis clínicas na capacidade funcional de mulheres em tratamento quimioterápico. Esc. Anna Nery. 2015; 19: 571-577.

14. Silva CB., Albuquerque V., Leite J. Qualidade de Vida em Pacientes Portadoras de Neoplasia Mamária Submetidas a Tratamentos Quimioterápicos. Revista Brasileira de Cancerologia. 2010; 56: 227-236.

15. Marupudi NI., Han JE., Li KW., Renard VM., Tyler BM., Brem H. Paclitaxel: a review of adverse toxicities and novel delivery strategies. Expert Opin Drug Saf. 2007; 6:.609-621 